\title{
Voluntary triggering of the first target attenuates the attentional blink
}

\author{
Ken Kihara • Jun I. Kawahara
}

Published online: 25 October 2011

(C) Psychonomic Society, Inc. 2011

\begin{abstract}
The term attentional blink (AB) refers to a failure in identifying the second of two targets, separated by less than $500 \mathrm{~ms}$, embedded in a rapid succession of nontargets. To examine whether the expectation of the onset of the first target affects the $\mathrm{AB}$, we compared the magnitudes of the $\mathrm{AB}$ deficit when participants triggered the appearance of the first target and when the target was presented automatically at some time point, as in traditional AB studies. In Experiment 1 , the first target appeared immediately after a participant's voluntary keypress, revealing that the accuracy for identifying the first target increased and that the $\mathrm{AB}$ deficit was attenuated. In Experiment 2, the temporal delay between a voluntary keypress and the first-target presentation was manipulated. The results showed that both targets could be reported accurately only when the first target was presented within $300 \mathrm{~ms}$ after the action. In Experiment 3, we ruled out an alternative explanation that would attribute the facilitation effect to mere physical movement, by examining the accuracy of target identification when participants voluntarily pressed a key but that action was unrelated to the onset of the first target. Taken together, the results suggest that voluntary action to trigger the onset of a visual target facilitates processing and reduces the subsequent $\mathrm{AB}$ deficit when the target appears within $300 \mathrm{~ms}$ of the action.
\end{abstract}

\section{K. Kihara $(\bowtie)$}

Kagoshima University,

1-21-40 Korimoto,

Kagoshima 890-0065, Japan

e-mail: kihara@ibe.kagoshima-u.ac.jp

K. Kihara J. I. Kawahara

National Institute of Advanced Industrial Science and Technology,

Central 6, 1-1-1 Higashi,

Tsukuba 305-8566, Japan

J. I. Kawahara

e-mail: jun.kawahara@aist.go.jp
Keywords Attentional blink - Cognitive and attentional control

We need to be aware of many transient changes in our daily lives, and given our dynamic environment, we need to attend selectively to critical signals to achieve our behavioral goals. In such circumstances, the visual system serves an essential function by selectively attending over time. Studies investigating the nature of selection across time have demonstrated selection failures using a variety of tasks, such as rapid serial visual presentation (RSVP), in which visual items are presented sequentially at the same location for about $100 \mathrm{~ms}$ each (Broadbent \& Broadbent, 1987). For example, Raymond, Shapiro, and Arnell (1992) revealed that when two targets were embedded in an RSVP stream and the stimulus onset asynchrony (SOA) of the two targets was less than $500 \mathrm{~ms}$, the identification of the first target impaired second-target processing. This phenomenon, called an attentional blink ( $\mathrm{AB})$, has been taken as a reflection of a fundamental characteristic of visual attention subsequent to focusing on an object (i.e., the first target), and is thus regarded as a clue for understanding the nature of selection across time (Shapiro, Arnell, \& Raymond, 1997).

Among the various factors that modulate the magnitude of the $\mathrm{AB}$, a temporal expectation of target onset has been shown to reduce the $\mathrm{AB}$ deficit. For example, when the second target and its directly preceding distractor shared the same color, the magnitude of the $\mathrm{AB}$ was reduced (Nieuwenstein, Chun, van der Lubbe, \& Hooge, 2005). This finding implies that participants expected the onset of the second target because the appearance of the distractor acted as a visual feature cue. As another example, Martens and Johnson (2005) demonstrated that the AB deficit was attenuated by a temporal cue indicating when the second 
target would appear. In their study, accurate identification of the second target increased when participants were informed of the SOA between the two targets by a cue presented at the beginning of each trial. In contrast, attenuation of the $\mathrm{AB}$ deficit did not occur when the cue was omitted but the SOA was fixed throughout a block. Although such consistency could be potentially useful for signaling the onset of the second target, no advantage of this consistency was observed. Martens and Johnson argued that an explicit cue enables participants to predict when the second target will appear, and thus attention can efficiently be allocated to targets appearing temporally adjacent to one another. As a result, targets can easily be distinguished from distractors, leading to correct reporting of the second target. However, these studies did not address whether providing a temporal cue that signals the onset of the first target but not of the second target would decrease the magnitude of the $A B$, even though major $A B$ models assume that the $A B$ deficit is triggered by the attentional processing of the first target (for recent reviews, see Dux \& Marois, 2009; Martens \& Wyble, 2010).

Recently, Olivers and Van der Burg (2008) examined the effect of temporal cuing of the first target in an AB-like context. In their study, a brief tone was presented during a relatively sparse stream of visual items $(250 \mathrm{~ms}$ per item). The researchers found that the magnitude of the $\mathrm{AB}$ was reduced by a tone coincident with the onset of the second target. However, such a reduction effect on the AB was not obtained by cuing the first target, although first-target accuracy was improved. Similarly, a tone presented with an item directly preceding the first target (i.e., $250 \mathrm{~ms}$ before the first target) did not reduce the $A B$, suggesting that a tone contingent on a target automatically enhances target processing.

To summarize, previous studies have demonstrated no reduction in the magnitude of the $\mathrm{AB}$ by first-target cuing (Olivers \& Van der Burg, 2008), whereas a substantial reduction has been found due to second-target cuing (Martens \& Johnson, 2005; Olivers \& Van der Burg, 2008). For the following reasons, however, it is premature to conclude that cuing the first target exerts no influence on the $\mathrm{AB}$ deficit. First, the first-target cuing effect could be short-lived and decay quickly. Because the items in the study by Olivers and Van der Burg were presented relatively sparsely (4 items per second), the transient dynamics of any cuing effect during a brief period should be examined with much finer temporal resolution, such as at a standard RSVP rate of 10 items per second. Second, the lack of reduction in the $\mathrm{AB}$ deficit by first-target cuing could be attributable to the temporal precision of the cue. Given that the perception of audio-visual synchrony is poor (Fujisaki \& Nishida, 2005), it is reasonable to assume that the cognitive system does not necessarily benefit from the information provided by the cuing. We assume that a similar problem would occur with any external cues, because they inherently require interpretation that demands additional processing. Specifically, visually presented cues, such as a square surrounding a target, require approximately $100 \mathrm{~ms}$ to be effective in a visual search task (Müller \& Rabbitt, 1989; Nakayama \& Mackeben, 1989) and in detection/identification tasks in RSVP streams (Weichselgartner \& Sperling, 1987).

One approach that potentially allows participants to anticipate the timing of the stimulus presentation involves the voluntary triggering of stimulus onset. Previous studies have shown that a visual stimulus triggered by a participant's action affected the event-related brain potentials involved in visual attention (Hughes \& Waszak, 2011: Nittono, Hamada, \& Hori, 2003). Specifically, when the appearance of the stimulus was triggered by a voluntary action, a positive brain potential component with a short latency (approximately $100 \mathrm{~ms}$ ) was observed (Hughes \& Waszak, 2011). The observation of a component that reflects the temporal aspect of visual attention (Hughes \& Waszak, 2011) suggests that voluntary triggering offers an opportunity to investigate whether the temporal expectation of a stimulus presentation affects visual attention.

Therefore, we introduced a new type of cuing procedure that involved the voluntary triggering of the first target to maximize the temporal informativeness of cuing. Participants in the present study determined the timing of each event (i.e., target onset) themselves, and thus were able to predict when the target would appear (Hommel, Müsseler, Aschersleben, \& Prinz, 2001; Stock \& Stock, 2004). This procedure avoids the need to interpret external cues, which previous studies required by using such methods as presenting distractors that shared colors with targets (Nieuwenstein et al., 2005), arranging fixed intertarget lags (Martens \& Johnson, 2005), or cuing the onset of a target with brief tones (Olivers \& Van der Burg, 2008). In the present study, we examined whether an expectation of first-target onset would reduce $\mathrm{AB}$ magnitude when the first target was produced by participants' voluntary actions.

\section{Experiment 1}

In Experiment 1, we compared the accuracy of target identification under the following two conditions: the selftriggering (self) condition, in which the first target appeared immediately after participants voluntarily pressed a designated key, and the control (automatic) condition, in which the first target appeared automatically with the same timing that had been produced by the same participant in the previous self condition (see the Method section for details). To increase the temporal sensitivity for detecting attentional modulation by the first-target cue, we presented items at a 
rate of $10 \mathrm{items} / \mathrm{s}$. We postulated that if the expectation of the first-target onset temporarily modulated the allocation of attention, the accuracy of target identification would be improved under the self condition relative to the automatic condition.

\section{Method}

Participants A group of 20 adults (11 male, 9 female, mean age 20.9 years) from the participant pool of the National Institute of Advanced Industrial Science and Technology (AIST) took part for payment. All had self-reported normal or corrected-to-normal vision.

Stimuli and apparatus The stimuli were presented on a 17in. computer monitor driven at a $100-\mathrm{Hz}$ refresh rate and controlled by MATLAB with the Psychophysics Toolbox (Brainard, 1997; Pelli, 1997). The viewing distance was $57 \mathrm{~cm}$. A stream of visual items consisted of two targets and 10-109 distractors. The distractors were randomly chosen digits (1-9), with a constraint that the same digit was not presented successively. The targets were two letters selected randomly from all of the letters of the English alphabet except I, O, and Q. Identical targets were never presented in a single trial. Each item subtended a visual angle of approximately $1^{\circ} \times 1^{\circ}$ and was presented for $100 \mathrm{~ms}$ with no interstimulus interval. The items were presented in dark gray $\left(1.2 \mathrm{~cd} / \mathrm{m}^{2}\right)$ against a black background $\left(0.3 \mathrm{~cd} / \mathrm{m}^{2}\right)$.

Procedure Two within-participants factors were examined: first-target volition (self vs. automatic) and intertarget SOA $(100,300,500$, or $700 \mathrm{~ms})$. Each volition condition was blocked and repeated alternately five times. Each block consisted of 32 trials (8 trials for each of the four SOA conditions) presented in random order. A total of 320 trials were given to each participant ( 4 SOAs $\times 2$ volition conditions $\times 40$ repetitions). Before the experimental session, participants engaged in 8 trials of a practice block and 32 trials of a filler self-condition block (identification performance in these trials was excluded from the analyses). In every trial during this filler self-condition block, the time at which participants pressed the space bar was recorded in order to present the first target at the same time in the subsequent auto-condition block. In the first experimental block, the automatic condition, in which the first target was presented in the same frame as in the filler self-condition block, was presented to one-half of the participants after the filler self-condition block. The other half of the participants started with the filler self-condition block, which was followed by an experimental self-condition block.

At the beginning of each block, a written instruction relating to volition was displayed. Each trial began with a hash mark (\#) presented for 1,000-3,000 ms to assist in fixation, followed by an RSVP stream. Under the self condition, participants were asked to press the space bar voluntarily within $10 \mathrm{~s}$ of the beginning of the RSVP stream to present the first target. If participants pressed the space bar, the next item was replaced by the first target (i.e., the first target was presented within $100 \mathrm{~ms}$ of the keypress). The appearance of the first target was contingent on the press, not the release, of the key. If participants did not press the space bar, the first target appeared automatically (i.e., the 101st item was the first target in this case). Under the automatic condition, participants were instructed to withhold pressing the space bar because the first target would appear automatically. The first target was presented in the same frame that had been recorded during the immediately preceding self-condition block (trial order was determined randomly). Within each stream, the first target was followed by nine digits and a letter that was the second target and was presented at one of the four randomly determined SOAs (i.e., the second target was the first, third, fifth, or seventh item after the first target). Once all of the items in a stream were presented, participants identified two target letters by pressing two corresponding keys. A warning message was presented when participants failed to withhold/ perform a response under the automatic/self condition. Each participant was allowed to respond at his or her own pace.

\section{Results}

In this and subsequent experiments, trials in which participants failed to withhold/perform a response to initiate the first target were excluded from further analyses $(0.2 \%$, $0.2 \%$, and $2 \%$ of the total trials for Exps. 1, 2, and 3, respectively). On average, participants pressed the space bar when 7.80 (SD 3.16) items had been presented. Identification responses were scored as correct regardless of report order. Accuracy in identifying the second target was based only on those trials in which the first target had been correctly identified. The accuracy rates for target identification are shown in Fig. 1 as a function of SOA conditions for both target-onset conditions.

A two-way repeated measures ANOVA conducted on the first-target accuracy revealed a significant main effect of volition, $F(1,19)=10.13, p<.01, \eta_{\mathrm{p}}^{2}=.35$, suggesting that the voluntarily triggered first target was $3.4 \%$ more likely to be reported correctly than was a first target presented automatically. The main effect of SOA was also significant, $F(3,57)=31.60, p<.001, \eta_{\mathrm{p}}^{2}=.62$. Post-hoc analyses (Tukey's HSD, $p<.05$ ) indicated that the firsttarget accuracies under the 100-ms SOA condition were $6.7 \%, 7.5 \%$, and $7.3 \%$ lower than those under the 300 -, $500-$, and 700-ms SOA conditions, respectively. The interaction between target onset and SOA was not significant, $F(3,57)=1.08$, n.s., $\eta_{\mathrm{p}}^{2}=.05$. 


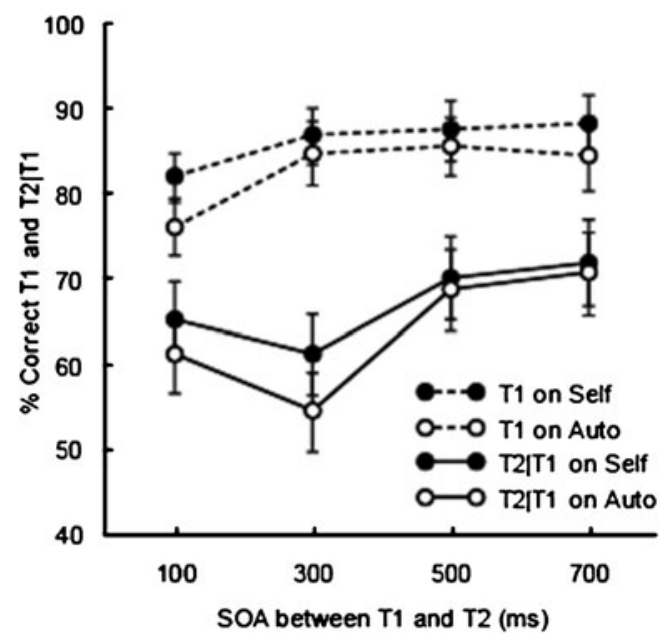

Fig. 1 Results of Experiment 1: Mean correct reports of the first target (T1) and of the second target given correct report of the first (T2|T1) as a function of SOA condition for both target-onset conditions. Error bars indicate the standard errors of the means across participants

A two-way repeated measures ANOVA conducted on second-target accuracy yielded significant main effects of volition, $F(1,19)=24.92, p<.001, \eta_{\mathrm{p}}^{2}=.57$, and SOA, $F(3,57)=16.12, p<.001, \eta_{\mathrm{p}}^{2}=.46$. Importantly, the interaction between these factors was also significant, $F(3$, $57)=3.16, p<.05, \eta_{\mathrm{p}}^{2}=.14$. To explore the nature of this interaction, we conducted post-hoc analyses (Tukey's HSD, $p<.05)$. First, we assessed whether the $\mathrm{AB}$ effect was reliable by comparing the performance on the second target at the 300-ms SOA to that at each of the other SOAs under each volition condition. The analyses indicated that the $\mathrm{AB}$ effects were significant under both the self (performance at the $300-\mathrm{ms}$ SOA was $8.9 \%$ and $10.7 \%$ worse than at the 500- and 700-ms SOAs, respectively, but did not differ significantly from performance at the 100-ms SOA) and automatic (performance at the 300 -ms SOA was $6.6 \%$, $14.2 \%$, and $16.2 \%$ worse than at the $100-, 500-$ and $700-\mathrm{ms}$ SOAs, respectively) conditions. These results indicate that the $A B$ occurred under both the self and automatic conditions in Experiment 1. Second, the effect of volition was tested by comparing performance for the second target under the self and the automatic conditions in each SOA condition. Post-hoc analysis (Tukey's HSD, $p<.05$ ) also indicated that the accuracy of identifying the second target at the 300-ms SOA was $6.7 \%$ higher under the self than under the automatic condition. However, we found no significant differences in second-target performance under the 100-, 500-, and 700-ms conditions. In addition, we analyzed the magnitude of the $\mathrm{AB}$ deficit by subtracting second-target accuracy in the 300-ms SOA condition from that accuracy in the 700-ms condition for a clearer test of the volition effect. A two-tailed $t$ test revealed that the magnitude of the $\mathrm{AB}$ deficit was $8.1 \%$ smaller for the self condition than for the automatic condition, $t(19)=3.11$, $p<.01$. These results suggest that the $\mathrm{AB}$ deficit was attenuated when the first target was voluntarily produced.

\section{Discussion}

The results of Experiment 1 suggest that identification of the first target was facilitated if observers could predict the exact time of the first target's onset by voluntary triggering. This was also true for the second target: Second-target identification was more accurate under the self condition than under the automatic condition. That is, the $\mathrm{AB}$ deficit was reduced under the self condition. Note that the results of Experiment 1 suggest that accuracy in identifying the first target at the SOA of $100 \mathrm{~ms}$ was lower than under the other SOA conditions. This could be attributed to competition between the two targets for limited attentional resources (Hommel \& Akyürek, 2005; Kawahara \& Enns, 2009; Potter, Staub, \& O'Connor, 2002).

The results of Experiment 1 demonstrate that accuracy in identification of the first target increased when the first target was voluntarily produced. Additionally, the second target presented during the $\mathrm{AB}$ period was also more likely to be reported correctly in the same condition. These results suggest that the expectation of the first target's onset due to its voluntary triggering modulates temporal selective attention. This new aspect of selective attention raises a further question regarding the duration of the effect of this expectation. In other words, we questioned whether the expectation could facilitate identification of the RSVP targets if a temporal delay intervened between the voluntary action triggering the first target and the target's appearance. In Experiment 2, we examined the effect of expectation on first- and second-target accuracies by manipulating the temporal delay between the voluntary keypress to initiate first-target onset and actual first-target presentation.

\section{Experiment 2}

Method

Participants A group of 32 adults (14 male, 18 female, mean age 24.0 years) from the participant pool of AIST took part for payment. All had self-reported normal or corrected-to-normal vision.

Stimuli and procedure The stimuli and procedures were the same as those used in Experiment 1, except for the following changes. The RSVP stream consisted of 209 (or fewer) distractors and two targets. Three within-participants factors were manipulated: volition (self or automatic), SOA 
between the two targets (300 or $700 \mathrm{~ms}$ ), and temporal delay between the keypress and the onset of the first target $(0-100,200-300,500-600$, or $1,000-1,100 \mathrm{~ms}$ after the keypress; i.e., 0, 2, 5, or 10 items appearing in between). Each temporal-delay condition was blocked and repeated twice. The block order was randomized across participants. Each temporal-delay block consisted of self and automatic subblocks. Each subblock consisted of 32 trials (2 SOAs $\times$ 16 repetitions) presented in a random order. Throughout the experiment, the self and automatic subblocks were presented alternately, and a written instruction regarding volition was displayed at the beginning of each subblock. In total, participants received 512 trials $(2$ SOAs $\times 2$ volitions $\times 4$ temporal delays $\times 32$ repetitions).

Results and discussion

The mean numbers of items presented before first-target onset (with $S D \mathrm{~s}$ ) were 8.08 (4.50), 11.66 (6.10), 14.67 (5.39), and 21.58 (9.03) for the 0 - to $100-, 200$ - to $300-$, 500 - to $600-$, and $1,000-$ to $1,100-\mathrm{ms}$ temporal-delay conditions, respectively. Second-target accuracies were based only on those trials in which the first target had been correctly identified. The accuracies of target identification are shown in Fig. 2 as a function of the volition, SOA, and temporal-delay conditions.

A three-way repeated measures ANOVA conducted on first-target accuracy indicated a significant main effect of volition, $F(1,31)=6.46, p<.05, \eta_{\mathrm{p}}^{2}=.17$, and a significant interaction between volition and temporal delay, $F(3,93)=3.70, p<.05, \eta_{\mathrm{p}}^{2}=.11$. Post-hoc analyses (Tukey's HSD, $p<.05$ ) indicated that the first-target accuracies at the 0 - to $100-\mathrm{ms}$ and 200 - to $300-\mathrm{ms}$ delays were $2.9 \%$ and $2.7 \%$ higher under the self condition than under the automatic condition. No other main effect or interaction was significant (all $F \mathrm{~s}<1.71$, n.s., all $\eta_{\mathrm{p}}^{2} \mathrm{~s}<.06$ ). These results suggest that first-target accuracy was increased when the target was presented within approximately $300 \mathrm{~ms}$ of the voluntary triggering of the target by a keypress.

A three-way repeated measures ANOVA conducted on second-target accuracies indicated significant main effects of volition, $F(1,31)=4.35, p<.05, \eta_{\mathrm{p}}^{2}=.12$, SOA, $F(1$, $31)=34.23, p<.001, \eta_{\mathrm{p}}^{2}=.52$, and temporal delay, $F(3$, $93)=6.38, p<.001, \eta_{\mathrm{p}}^{2}=.17$. Additionally, a significant three-way interaction among volition, SOA, and temporal delay was found, $F(3,93)=2.86, p<.05, \eta_{\mathrm{p}}^{2}=.08$. Posthoc analysis (Tukey's HSD, $p<.05$ ) revealed that accuracy in identifying the second target at the $300-\mathrm{ms}$ SOA was higher under the self condition than under the automatic condition for the 0 - to $100-\mathrm{ms}$ and 200 - to $300-\mathrm{ms}$ temporal delays (5.4\% and 5.8\%, respectively), but not for the 500 to $600-$ and $1,000-$ to $1,100-\mathrm{ms}$ temporal delays. There were no significant differences in second-target performance between the 700-ms SOA condition and the other SOA conditions. No other main effect or interaction was significant (all $F_{\mathrm{s}}<2.71$, n.s., all $\eta_{\mathrm{p}}^{2} \mathrm{~s}<.09$ ). These results suggest that the $A B$ deficit was attenuated when the temporal delay between the onset of the first target and the voluntary keypress was within $300 \mathrm{~ms}$.

Replicating Experiment 1, the results of Experiment 2 indicate that not only the first target but the second was reported more accurately if the second target followed the first by $300 \mathrm{~ms}$ and if the first target appeared directly after the performance of a voluntary action. Additionally, identification of the targets was facilitated when the first target was presented 200-300 ms after the voluntary action. However, if the temporal delay between the voluntary action and the first target was longer than $500 \mathrm{~ms}$, the facilitation effect disappeared. These results indicate that the expectation of the stimulus onset, driven by the voluntary action, enhanced temporal attention for approximately $300 \mathrm{~ms}$ after that action.

\section{Experiment 3}

The results so far suggest that processing of the first target is enhanced if participants can anticipate the onset of the target and if it appears within $300 \mathrm{~ms}$ of the voluntary keypress. Additionally, the $\mathrm{AB}$ deficit appears to be attenuated under these conditions. On the basis of these findings, we argue that the expectation of stimulus onset facilitated temporal selective attention for up to $300 \mathrm{~ms}$. This facilitation reduced the processing load for the first target, and as a result, a reduction in the $\mathrm{AB}$ deficit ensued. Alternatively, however, one could argue that the facilitation of the first-target identification was not involved with the expectation of the onset of the first target. Rather, the physical movement involved in the keypress could modulate temporal attention. Although there is no conclusive evidence for this kind of relationship between physical movement and cognitive processing, several studies have suggested that cognitive performance may be improved by performing a sensory-motor task (McMorris \& Graydon, 2000), potentially due to an increase in arousal (Kahneman, 1973). To rule out this possibility, in Experiment 3, we examined whether accurate identification of RSVP targets would be improved if the onset of the first target coincided with keypresses that were unrelated to the onset of the first target.

Method

Participants A group of 26 adults (24 males, 2 females, mean age 21.4 years) from the participant pool of AIST 
Fig. 2 Results of Experiment 2: Mean correct reports of the first target (T1) and of the second target given correct report of the first (T2|T1) as a function of the SOA between the first and second targets. Each panel in the figure shows the results from each temporal-delay condition. Error bars indicate the standard errors of the means across participants
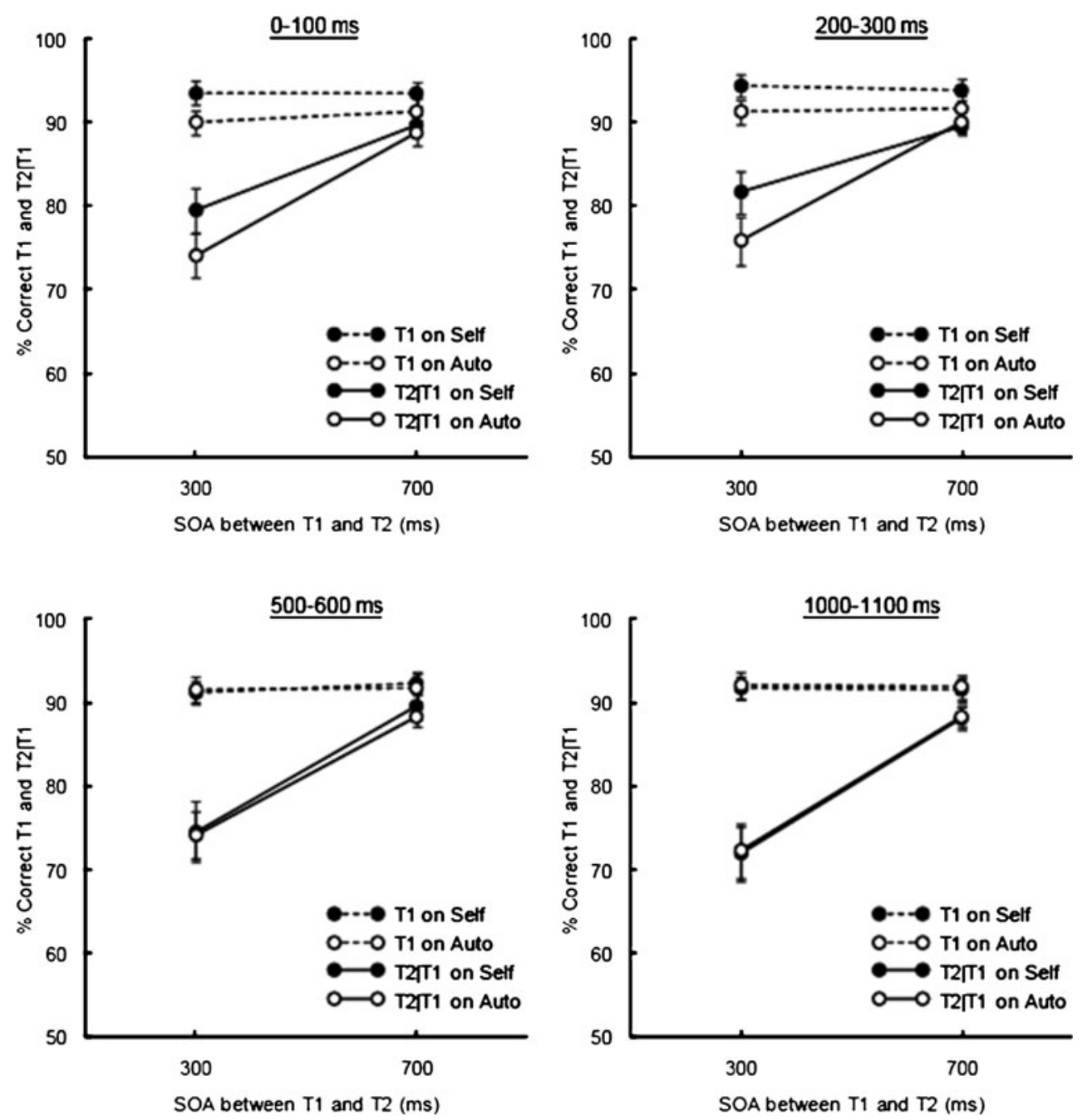

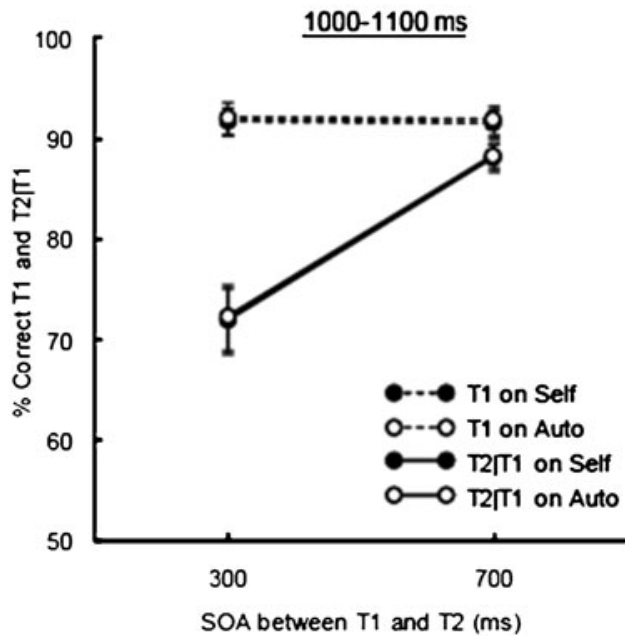

took part for payment. All had self-reported normal or corrected-to-normal vision.

Stimuli and procedure The stimuli and procedures were the same as those used in Experiment 1, except for the following changes. The RSVP stream consisted of 34 distractors and two targets. The SOA between the two targets was manipulated as a within-participants variable (300 or $700 \mathrm{~ms}$ ). Under each SOA condition, the first target was presented in the sequence somewhere between the third and the 26th items. Participants received a total of 480 trials $(2$ SOAs $\times 24$ temporal locations of the first target $\times 10$ repetitions), preceded by 8 practice trials. In Experiments 1 and 2, participants pressed the space bar more than $3 \mathrm{~s}$ after the initiation of the stream in only a few trials (1.7\% in Exp. 1 and 5.4\% in Exp. 2). Therefore, in Experiment 3, participants were instructed to press the space bar voluntarily within $3 \mathrm{~s}$ after the RSVP stream had started and to identify the two targets. They were informed that the keypress was unrelated to any events in the RSVP stream.

\section{Results and discussion}

On average, participants pressed the space bar when 7.55 (SD 1.43) items had been presented. We compared the differences in identification accuracy as a function of the temporal delay between the keypress and the onset of the first target by sorting trials retrospectively. We chose trials in which the temporal delay ranged from -200 to $1,100 \mathrm{~ms}$, where the negative value means that the onset of the first target preceded the keypress. Other trials were omitted because the number of corresponding trials was too small (fewer than four trials). Data from 6 participants were excluded from further analysis because the number of trials on which the first target was correctly identified was too small (fewer than four trials) in at least one of the temporaldelay conditions, leaving a total of 20 participants. 
Fig. 3 Results of Experiment 3: Mean correct reports of the first target (T1) and of the second target given correct report of the first (T2|T1) as a function of the temporal delay between the keypress and the onset of the first target. Error bars indicate the standard errors of the means across participants

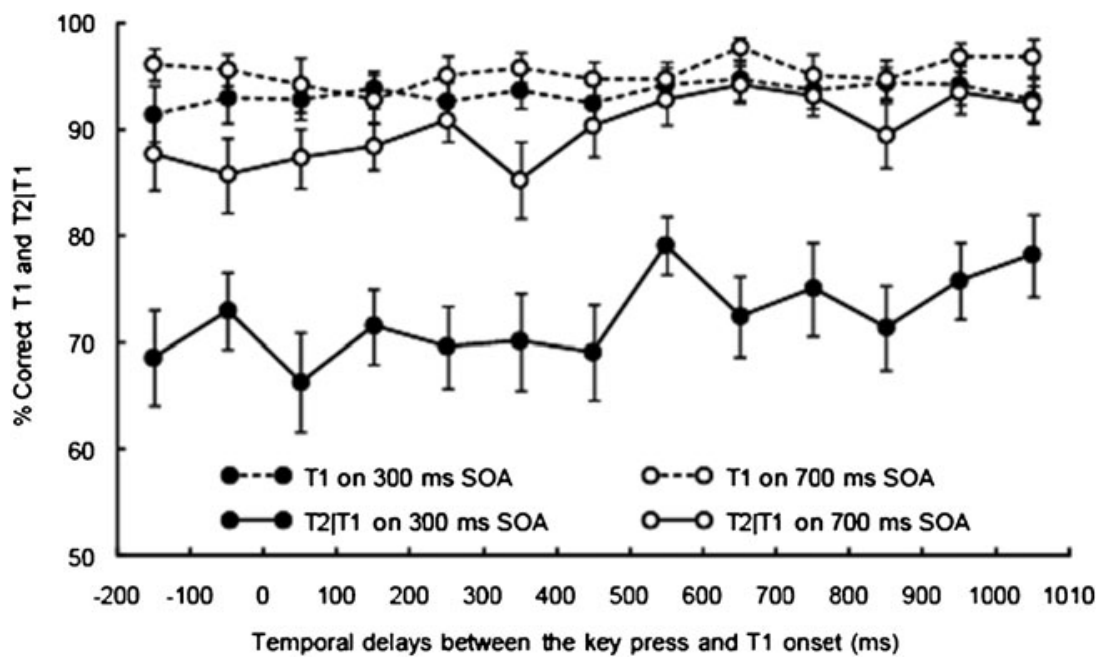

Accuracy for the first and second targets is shown in Fig. 3 as a function of SOA and the temporal delay between the keypress and the onset of the first target.

As can be seen in Fig. 3, the first-target accuracy when the SOA was 300 ms was $2.3 \%$ lower than when the SOA was $700 \mathrm{~ms}$. This difference was confirmed by a two-way repeated measures ANOVA as a significant main effect of SOA, $F(1,19)=10.72, p<.001, \eta_{\mathrm{p}}^{2}=.36$. However, the ANOVA found no significant main effect of temporal delay, $F(12,228)=0.77$, n.s., $\eta_{\mathrm{p}}^{2}=.04$, and no interaction between SOA and temporal delay, $F(12,228)=0.76$, n.s., $\eta_{\mathrm{p}}^{2}=.04$.

$A$ robust $\mathrm{AB}$ deficit was obtained - that is, the secondtarget accuracy was $19.6 \%$ lower in the $300-\mathrm{ms}$ SOA condition than in the 700-ms SOA condition, as indicated by a significant main effect of SOA, $F(1,19)=49.24, p<.001$, $\eta_{\mathrm{p}}^{2}=.72$, in a two-way repeated measures ANOVA conducted on second-target accuracy. However, there was no significant main effect of the temporal delay, $F(12,228)=$ 1.35 , n.s., $\eta_{\mathrm{p}}^{2}=.07$, and no interaction between SOA and the temporal delay, $F(12,228)=0.98$, n.s., $\eta_{\mathrm{p}}^{2}=.05$.

A number of issues are raised by the present results. First, the finding that the accuracy for the first target was slightly higher at the 700-ms SOA than at the 300-ms SOA could be attributed to intertarget interference (Potter et al., 2002). Such interference would continue even if the SOA were over $100 \mathrm{~ms}$ (e.g., Exp. 1 of Chun \& Potter, 1995; Exp. 2 of Hommel \& Akyürek, 2005). Second, regarding the temporal delay between the voluntary keypress and the onset of each target, this variable had no impact on the magnitude of the AB, and similarly, the delay had no effect on first-target accuracy. These results are inconsistent with the idea that a mere voluntary keypress action is sufficient to enhance visual identification, even when the keypress does not trigger the onset of the target. Rather, the present results support the idea that the facilitation of target identification observed in Experiments 1 and 2 was due to the expectation of the onset of the first target.

\section{General discussion}

The purpose of the present study was to investigate how the expectation of target onset modulates temporal selective attention as indexed by the $\mathrm{AB}$ deficit. The present results are the first to provide direct evidence of the time course of the expectation effect's facilitating the processing of two targets embedded in an RSVP stream. A previous study demonstrated that expecting the onset of the second target attenuated the magnitude of the AB deficit (Martens \& Johnson, 2005). Given that the processing of the first target determines the occurrence of the AB (Dux \& Marois, 2009; Martens \& Wyble, 2010; Shapiro et al., 1997), it is reasonable to assume that expecting the onset of the first target would facilitate the processing of the first target and that the magnitude of the $\mathrm{AB}$ deficit would decrease as a result. However, no direct supporting evidence has been found in experiments in which the onset of the first target was associated with an arbitrary external cue (e.g., a tone; Olivers \& Van der Burg, 2008). In the present study, we used the voluntary triggering of the first target to maximize the utility of the likelihood of target appearance while minimizing the time required for interpreting this external cue (Hommel et al., 2001; Stock \& Stock, 2004).

The results of Experiment 1 revealed that the expectation of the onset of the first target facilitated identification of the target when it directly followed the voluntary keypress. The magnitude of the AB deficit was also attenuated accordingly. Experiment 2 demonstrated that this facilitation was short-lived: The expectation effect was observed only when 
the first target was presented within $300 \mathrm{~ms}$ of its voluntary triggering. In Experiment 3, we ruled out an alternative explanation that would attribute the facilitation effect to mere physical movement by demonstrating that the accuracy of target identification was unaffected by a voluntary keypress when that action was unrelated to the onset of the first target.

One might wonder whether the accuracy with which the second target would be identified would vary according to when participants triggered the appearance of the first target. In fact, no such variations were found. The mean intraindividual correlations between second-target accuracy and keypress latency from the beginning of the RSVP stream were very low $(r=-.10$ to .09 under all conditions in Exps. 1 and 2). It was also unlikely that the second-target accuracy of participants who tended to trigger the first target early differed from that of participants who triggered it late, because no significant correlations were found between second-target performance and keypress latency in individual participants $(r=-.21$ to .25 under all conditions in Exps. 1 and 2, all $p s>.24)$. We could not find any evidence suggesting that the second-target performance was affected by the delay of the voluntary triggering of the first target's onset.

In Experiments 1 and 2, when the first target appeared within $300 \mathrm{~ms}$ of the voluntary keypress, the identification accuracy for both the first and second targets (presented during the $\mathrm{AB}$ period) increased. These results are apparently inconsistent with the findings in Olivers and Van der Burg's (2008) study; they found that a tone accompanying the first target improved only the firsttarget accuracy. This inconsistency could be attributed to the specificity of audio-visual binding, such that a tone presented with a visual target segregates the target from other visual stimuli (Vroomen \& de Gelder, 2000). According to this view, a tone presented with the first target separates the processing of the first target from that of the second target, resulting in an increase in first-target accuracy without any effect on the identification of the second target. In contrast, the voluntary triggering of a visual target does not induce such a segregation effect.

It has been argued that temporal attention is modulated when the onset of a stimulus can be predicted by the voluntary triggering method (Hughes \& Waszak, 2011; see also Correa, Lupiáñez, Madrid, \& Tudela, 2006; Miniussi, Wilding, Coull, \& Nobre, 1999). Therefore, a first target triggered by a voluntary action would affect temporal attention, leading to the reduction in the $\mathrm{AB}$ deficit observed in this study. However, the results of Experiment 2 suggest that prediction of the onset of the first target does not modulate temporal attention during an entire trial. Instead, its facilitative effect on the identification of the target was limited to only those trials on which the first target appeared within $300 \mathrm{~ms}$ of the voluntary keypress. We argue that this limitation is attributable to the interaction between the facilitative effect and the mechanisms involved in the subsequent $\mathrm{AB}$.

In Experiment 1, we observed an attenuation of the $\mathrm{AB}$ that was associated with improved identification of the first target. There are two possible explanations for these results. One is that enhancement of first-target processing contributed to the attenuation of the AB. In this case, the expectation of the first target's onset enhanced first-target processing itself (Niemi \& Nätänen, 1981). As a result, second-target processing was facilitated. That is, the expectation of the first target's onset indirectly facilitated processing of the second target via reduction of the processing load for the first target. A second explanation is that both first- and second-target processing benefited independently from the expectation. If the expectation of the first target's onset transiently improved general attentional resources, identification accuracy for both the first and second targets presented during the $\mathrm{AB}$ period would be enhanced. That is, the expectation of the first target's onset directly benefits the processing of both targets. The results of Experiment 2 support the former explanation. In Experiment 2, the identification accuracy of the second target presented $300 \mathrm{~ms}$ after the first target's onset during the $\mathrm{AB}$ period improved when the first target appeared 200-300 ms after the keypress. In this case, the second target was presented 500-600 ms after the keypress. If the increase in the second-target accuracy were due to a transient increment in attentional resources triggered by the expectation of the first target's onset, the processing of the first target would also be facilitated on trials in which the first target was presented $500-600 \mathrm{~ms}$ after the keypress. However, the identification accuracy of the first target presented 500-600 ms after the keypress was not enhanced. These results suggest that the benefit of expectation disappears within $500 \mathrm{~ms}$ of the keypress. Therefore, the second target presented 500-600 ms after the keypress did not benefit directly from the transient increment in attentional resources triggered by the expectation of the first-target onset. Rather, processing of the second target presented during the $\mathrm{AB}$ period was facilitated because the first target, presented before the benefit of the expectation decayed, was processed more efficiently.

The present finding regarding the effect of the expectation of the first target's onset on target processing is in line with current theories about the $\mathrm{AB}$ that have posited that the processing of the first target is determined by transient attention driven by the onset of the first target (Bowman \& Wyble, 2007; Olivers, 2007; Olivers \& Meeter, 2008; Wyble, Bowman, \& Nieuwenstein, 2009). In this view, transient attention develops rapidly, reaching a peak around $100 \mathrm{~ms}$ after its onset, and decays quickly (Müller \& Rabbitt, 1989; 
Nakayama \& Mackeben, 1989; Weichselgartner \& Sperling, 1987). If such transient attention were enhanced, the processing of the first target would be facilitated, leading to attenuation of the $\mathrm{AB}$ deficit. Therefore, we suggest that the expectation of the onset of the first target boosts the transient attention triggered by the expected onset. The present study demonstrated that the facilitation resulting from expectation is a transient phenomenon in that the effect decays by $500 \mathrm{~ms}$ or less after the voluntary action. In other words, voluntary production of the first target triggers transient attention. We tentatively assume that when the first target appears within about $500 \mathrm{~ms}$ of its voluntary production, the processing of that target is facilitated by volitional (i.e., transient attention triggered by the voluntary production) and external (i.e., the onset of the first target) triggers. Obviously, further studies will be required in order to test these possibilities.

In conclusion, the present study demonstrated that the voluntary action to trigger the onset of a visual target facilitated the processing of the target and reduced the subsequent attentional deficit (i.e., the $\mathrm{AB}$ deficit). This facilitation was observed only when the first target appeared within about $300 \mathrm{~ms}$ of the action. No such effect of the action was obtained when the action by itself contained no predictive information regarding the onset of the target. Thus, the expectation, not just the physical movement, enhanced temporal selective attention for approximately $300 \mathrm{~ms}$ after the voluntary action.

Author note This work was partially supported by grants from the Japan Society for the Promotion of Science to the authors.

\section{References}

Bowman, H., \& Wyble, B. (2007). The simultaneous type, serial token model of temporal attention and working memory. Psychological Review, 114, 38-70.

Brainard, D. H. (1997). The Psychophysics Toolbox. Spatial Vision, 10, 433-436. doi:10.1163/156856897X00357

Broadbent, D. E., \& Broadbent, M. H. P. (1987). From detection to identification: Response to multiple targets in rapid serial visual presentation. Perception \& Psychophysics, 42, 105-113. doi: 10.3758/BF03210498

Chun, M. M., \& Potter, M. C. (1995). A two-stage model for multiple target detection in rapid serial visual presentation. Journal of Experimental Psychology: Human Perception and Performance, 21, 109-127. doi:10.1037/0096-1523.21.1.109

Correa, Á., Lupiáñez, J., Madrid, E., \& Tudela, P. (2006). Temporal attention enhances early visual processing: A review and new evidence from event-related potentials. Brain Research, 1076, 116-128. doi:10.1016/j.brainres.2005.11.074

Dux, P. E., \& Marois, R. (2009). The attentional blink: A review of data and theory. Attention, Perception, \& Psychophysics, 71, 1683-1700. doi:10.3758/APP.71.8.1683

Fujisaki, W., \& Nishida, S. (2005). Temporal frequency characteristics of synchrony-asynchrony discrimination of audio-visual signals. Experimental Brain Research, 166, 455-464.
Hommel, B., \& Akyürek, E. G. (2005). Lag-1 sparing in the attentional blink: Benefits and costs of integrating two events into a single episode. Quarterly Journal of Experimental Psychology, 58A, 1415-1433.

Hommel, B., Müsseler, J., Aschersleben, G., \& Prinz, W. (2001). The Theory of Event Coding (TEC): A framework for perception and action planning. The Behavioral and Brain Sciences, 24, 849878. doi:10.1017/S0140525X01000103

Hughes, G., \& Waszak, F. (2011). ERP correlates of action effect prediction and visual sensory attenuation in voluntary action. NeuroImage, 56, 1632-1640.

Kahneman, D. (1973). Attention and effort. Englewood Cliffs, NJ: Prentice-Hall.

Kawahara, J., \& Enns, J. T. (2009). Selection difficulty and interitem competition are independent factors in rapid visual stream perception. Journal of Experimental Psychology: Human Perception and Performance, 35, 146-158.

Martens, S., \& Johnson, A. (2005). Timing attention: Cuing target onset interval attenuates the attentional blink. Memory \& Cognition, 33, 234-240. doi:10.3758/BF03195312

Martens, S., \& Wyble, B. (2010). The attentional blink: Past, present, and future of a blind spot in perceptual awareness. Neuroscience and Biobehavioral Reviews, 34, 947-957.

McMorris, T., \& Graydon, J. (2000). The effect of incremental exercise on cognitive performance. International Journal of Sport Psychology, 31, 66-81.

Miniussi, C., Wilding, E. L., Coull, J. T., \& Nobre, A. C. (1999). Orienting attention in time: Modulation of brain potentials. Brain, 122, 1507-1518.

Müller, H. J., \& Rabbitt, P. M. (1989). Reflexive and voluntary orienting of visual attention: Time course of activation and resistance to interruption. Journal of Experimental Psychology: Human Perception and Performance, 15, 315-330. doi:10.1037/ 0096-1523.15.2.315

Nakayama, K., \& Mackeben, M. (1989). Sustained and transient components of focal visual attention. Vision Research, 29, 16311647.

Niemi, P., \& Näätänen, R. (1981). Foreperiod and simple reaction time. Psychological Bulletin, 89, 133-162.

Nieuwenstein, M. R., Chun, M. M., van der Lubbe, R. H. J., \& Hooge, I. T. C. (2005). Delayed attentional engagement in the attentional blink. Journal of Experimental Psychology: Human Perception and Performance, 31, 1463-1475. doi:10.1037/00961523.31.6.1463

Nittono, H., Hamada, A., \& Hori, T. (2003). Brain potentials after clicking a mouse: A new psychophysiological approach to human-computer interaction. Human Factors, 45, 591599.

Olivers, C. N. L. (2007). The time course of attention: It is better than we thought. Current Directions in Psychological Science, 16, $11-$ 15.

Olivers, C. N. L., \& Meeter, M. (2008). A boost and bounce theory of temporal attention. Psychological Review, 115, 836-863. doi:10.1037/a0013395

Olivers, C. N. L., \& Van der Burg, E. (2008). Bleeping you out of the blink: Sound saves vision from oblivion. Brain Research, 1242, 191-199.

Pelli, D. G. (1997). The VideoToolbox software for visual psychophysics: Transforming numbers into movies. Spatial Vision, 10, 437-442. doi:10.1163/156856897X00366

Potter, M. C., Staub, A., \& O'Connor, D. H. (2002). The time course of competition for attention: Attention is initially labile. Journal of Experimental Psychology: Human Perception and Performance, 28, 1149-1162. doi:10.1037/0096-1523.28.5.1149

Raymond, J. E., Shapiro, K. L., \& Arnell, K. M. (1992). Temporary suppression of visual processing in an RSVP task: An attentional 
blink? Journal of Experimental Psychology: Human Perception and Performance, 18, 849-860. doi:10.1037/0096-1523.18.3.849

Shapiro, K. L., Arnell, K. M., \& Raymond, J. E. (1997). The attentional blink. Trends in Cognitive Sciences, 1, 291-296. doi:10.1016/S1364-6613(97)01094-2

Stock, A., \& Stock, C. (2004). A short history of ideo-motor action. Psychological Research, 68, 176-188.

Vroomen, J., \& de Gelder, B. (2000). Sound enhances visual perception: Cross-modal effects of auditory organization on vision. Journal of Experimental Psychology: Human Perception and Performance, 26, 1583-1590.

Weichselgartner, E., \& Sperling, G. (1987). Dynamics of automatic and controlled visual attention. Science, 238, 778-780. doi:10.1126/science. 3672124

Wyble, B., Bowman, H., \& Nieuwenstein, M. (2009). The attentional blink provides episodic distinctiveness: Sparing at a cost. Journal of Experimental Psychology: Human Perception and Performance, 35, 787-807. doi:10.1037/a0013902 